\title{
Spatial, temporal and spatio-temporal clusters of measles incidence at the county level in Guangxi, China during 2004-2014: flexibly shaped scan statistics
}

\author{
Xianyan Tang ${ }^{1,2^{*}+}$, Alan Geater ${ }^{2}$, Edward McNeil ${ }^{2}$, Qiuyun Deng ${ }^{3 \dagger}$, Aihu Dong ${ }^{3}$ and Ge Zhong ${ }^{3 *}$
}

\begin{abstract}
Background: Outbreaks of measles re-emerged in Guangxi province during 2013-2014, where measles again became a major public health concern. A better understanding of the patterns of measles cases would help in identifying high-risk areas and periods for optimizing preventive strategies, yet these patterns remain largely unknown. Thus, this study aimed to determine the patterns of measles clusters in space, time and space-time at the county level over the period 2004-2014 in Guangxi.

Methods: Annual data on measles cases and population sizes for each county were obtained from Guangxi CDC and Guangxi Bureau of Statistics, respectively. Epidemic curves and Kulldorff's temporal scan statistics were used to identify seasonal peaks and high-risk periods. Tango's flexible scan statistics were implemented to determine irregular spatial clusters. Spatio-temporal clusters in elliptical cylinder shapes were detected by Kulldorff's scan statistics. Population attributable risk percent (PAR\%) of children aged $\leq 24$ months was used to identify regions with a heavy burden of measles.
\end{abstract}

Results: Seasonal peaks occurred between April and June, and a temporal measles cluster was detected in 2014. Spatial clusters were identified in West, Southwest and North Central Guangxi. Three phases of spatio-temporal clusters with high relative risk were detected: Central Guangxi during 2004-2005, Midwest Guangxi in 2007, and West and Southwest Guangxi during 2013-2014. Regions with high PAR\% were mainly clustered in West, Southwest, North and Central Guangxi.

Conclusions: A temporal uptrend of measles incidence existed in Guangxi between 2010 and 2014, while downtrend during 2004-2009. The hotspots shifted from Central to West and Southwest Guangxi, regions overburdened with measles. Thus, intensifying surveillance of timeliness and completeness of routine vaccination and implementing supplementary immunization activities for measles should prioritized in these regions.

Keywords: Spatial, Temporal, Spatio-temporal, Irregular cluster, Measles, Scan statistics

\footnotetext{
* Correspondence: tangxianyan0746@163.com; gxzhongge@163.com

${ }^{\dagger}$ Equal contributors

${ }^{1}$ Department of Epidemiology and Biostatistics, School of Public Health,

Guangxi Medical University, Guangxi Zhuang Autonomous Region, China

${ }^{3}$ Guangxi Center for Disease Control and Prevention, Institute of Vaccination,

Guangxi Zhuang Autonomous Region, China

Full list of author information is available at the end of the article
} 


\section{Background}

Measles is a highly contagious disease and a leading cause of death among children worldwide [1]. More than 95\% of measles deaths have been reported in low-middle income countries and regions with resource-poor health facilities [2]. China, a middle-income country, is one of the member nations in the WHO Western Pacific Region, accounting for approximately $70 \%$ of the reported measles cases in this region since 2003 [3]. According to the schedule of expanded program on immunization (EPI) in China, routine measles vaccination covers two doses of measles-containing vaccine (i.e. MCV1, MCV2). Children should be vaccinated with MCV1 at the age of 8 months, and MCV2 should be given to children between the ages of 18 and 24 months. In addition, the current schedule of supplementary immunization activities (SIA) for measles is during September 11-20 per year. Despite the routine two-dose measles vaccination coverage across most regions of China being over 90\%, large-scale outbreaks of measles still occurred in Guangxi, Beijing, Zhejiang and Shangdong provinces [4-7].

Prior to 2003, Guangxi was one of the provinces in China with the highest measles incidence [8]. Since 2004, the incidence has dropped below the national average [8]. However, large-scale measles outbreaks reemerged in Guangxi during 2013-2014 [9]. Therefore, measles again became a major public health concern in Guangxi. To effectively improve the timeliness of routine measles vaccination and to implement supplementary immunization activities for measles in high-risk regions, a better understanding of the spatial, temporal and spatio-temporal patterns of measles is needed. Unfortunately, these patterns remain largely unknown in Guangxi.

Scan statistics, which are used in spatio-temporal analyses, have been widely applied to explore the patterns of disease and detect high-risk diseases clusters, particularly for diseases having limited resources for prevention [10]. However, most previous studies examined regular (i.e. circular or cylindrical shape) diseases clusters, which are unrealistic, since the true shapes of clusters are usually less regular [11-16]. Few studies have explored irregularly shaped diseases clusters, which might more realistically reflect the actual, albeit complicated, patterns of disease [17, 18]. Moreover, most studies have utilized the relative risk (RR) derived from scan statistics to identify high-risk regions $[11,13$, $14,16]$. Few studies have measured the population attributable risk percent (PAR\%) to evaluate the regional burden of disease [7]. Additionally, there is a paucity of studies on the spatio-temporal patterns of measles, particularly in Guangxi, a region with large-scale measles outbreaks.
Therefore, a spatio-temporal analysis was conducted to identify spatial, temporal and spatio-temporal irregular clusters of measles incidence at the county level in Guangxi between 2004 and 2014, integrating flexibly shaped scan statistics and PAR\%. Results of this study may not only help understand the spatio-temporal shift trend of measles in Guangxi, but also facilitate evaluating the geographical inequities in the burden of measles. Moreover, findings may provide essential evidence for implementing supplementary immunization activities for measles and strengthening the surveillance of timeliness and completeness of routine measles vaccination in overburdened regions, as well as other developing countries with a similar context.

\section{Methods}

Study area

The study site was Guangxi Zhuang Autonomous Region, a mountainous province in Southwest China. Geographically, Guangxi has proximity to the Association of Southeast Asian Nations (ASEAN) member states. It has a subtropical climate, with high humidity and temperature. Guangxi consists of 14 prefectures and 109 counties or urban districts, with an area of $236,700 \mathrm{~km}^{2}$ and a population of 46.8 million residents in 2012. In this study, a total of 109 counties or urban districts were included for spatio-temporal analysis.

\section{Data sources}

Yearly data on measles cases for each county between 2004 and 2014 was obtained from the National Notifiable Infectious Disease Surveillance System in Guangxi Center for Disease Control and Prevention. As a class B notifiable disease, all measles cases were laboratory confirmed by at least two experts according to the WHO manual for laboratory diagnosis of measles virus infection [19]. The annual population size and the proportion of the total number of children aged $\leq 24$ months for each county during 2004-2014 was obtained from Guangxi Bureau of Statistics. Based on the population size and the number of notifiable measles cases, the annual incidence (per 100,000 population) was calculated for each county. In addition, a vector map of Guangxi at the county level was collected from Guangxi Bureau of Surveying, Mapping and Geoinformation.

\section{Temporal, spatial and Spatio-temporal analysis}

An epidemic curve of monthly measles cases during January 2004 to December 2014 was drawn to reveal the seasonal peaks, and a trend line was fitted to identify the annual trend in incidence. Purely temporal clusters of high incidence were detected by Kulldorff's temporal scan statistics based on a discrete Poisson model in SaTScan 9.4.2 [10]. 
Inverse distance weighted interpolation of annual incidence was calculated in Arc GIS 10.0 to generate smoothed maps of measles incidence [20]. Spatial irregular clusters of high-incidence were tested by Tango's flexible spatial scan statistics in FleXScan 3.1.2, using a Poisson model with restricted log likelihood ratio (default alpha $=0.2$ ) and 15 census areas as the maximum spatial cluster size [21, 22]. These hotspots were visualized in Arc GIS 10.0.

Kulldorff's retrospective space-time scan statistics based on the Poisson model and elliptical-cylinder scanning window in SaTScan 9.4.2 was applied to detect irregular spatio-temporal clusters of high-incidence during 20042014, which were visualized in three-dimensions in AutoCAD $2010[10,18]$.

Generally, Kulldorff's retrospective scan statistics are used to detect high-risk clusters in either a purely temporal, purely spatial or jointly spatio-temporal setting, based on a gradual scanning window [10]. The scanning window is an interval for purely temporal scan statistics, and a circle or ellipse for purely spatial scan statistics. For retrospective spatio-temporal scan statistics, the scanning window is a cylindrical or ellipticalcylinder shape with a base corresponding to space and with height corresponding to time. The window moves over space and time scanning for elevated risks inside the window as compared to outside. For each change in space and time in the window, the log likelihood ratio (LLR), a statistic is automatically calculated. The window with the highest maximum LLR is deemed to be the most likely cluster. The $P$-value for clustering is calculated using Monte Carlo simulation.

Relative risk (RR) was determined in the spatiotemporal scan statistics analysis, the estimated risk within the cluster divided by the estimated risk outside the cluster. In this perspective, the reference group for calculating $R R$ is the estimated risk outside the cluster. Mathematically, RR is calculated as the observed cases divided by the expected cases within the cluster divided by the observed cases divided by the expected cases outside the cluster.

The elliptical-cylinder was the preferred shape for use during scanning to detect irregular clusters of spacetime in this study. The maximum spatial cluster size was set to $15 \%$ of the population at risk to avoid falsely absorbing neighborhood regions into an unrealistic cluster, and the maximum temporal cluster size was set to the default $50 \%$ of the scan timeframe [23]. For statistical power evaluation, the number of Monte Carlo replications was set to 999 . Temporal trend adjustment was conducted by log linear model with automatically calculated trend, and spatial trends were adjusted by nonparametric statistics with spatial stratified randomization. Clusters were reported without geographical overlap.

\section{Population attributable risk percent}

From a public health perspective, the population attributable risk percent (PAR\%) reflects the fraction of all cases in the population attributable to being in that cluster [24]. For disease burden analysis, we calculated PAR\% by county to estimate how much measles could be avoided if routine two-dose measles vaccination was timely and completely delivered to population aged $\leq 24$ months. The formula for PAR\% is listed as follows [7]:

$$
P A R \%=\frac{P_{E}(R R-1)}{P_{E}(R R-1)+1} \times 100
$$

where, RR is the relative risk of measles in each county, which was derived from the results of spatio-temporal scan statistics in SaTScan software, and $P_{\mathrm{E}}$ is the proportion of the population aged $\leq 24$ months in each county. As $P_{\mathrm{E}}$ can not be reduced, a reduction in PAR\% can be achieved only by reducing the RR. This requires an increased coverage of timely and complete measles vaccination in the county. Namely, the routine twodose measles vaccines should be delivered timely to children aged $\leq 24$ months in the county, according to the national immunization schedule of China [25].

\section{Linear directional mean}

The mean center of measles incidence is the geographic average location of a set of points. In this study, the mean centers of the locations of the counties having measles case were measured. And the mean centers denote the area and extent of the incidence of measles cases. The linear directional mean was conducted in Arc GIS 10.0 to measure the directional trend of measles transmission in Guangxi, using the annual mean center movements of measles incidence during 2004-2014 [26].

\section{Ethical considerations}

This study was approved by the Ethics Committees of Prince of Songkla University and Guangxi Medical University. Dataset of measles cases was approved by Guangxi Center for Disease Control and Prevention.

\section{Results}

\section{Purely temporal clusters of measles}

Figure 1 shows the monthly distribution of measles cases between 2004 and 2014 and the temporal trend in measles incidence. Prior to 2009, seasonal peaks occurred predominantly during April to June in Guangxi with one unseasonal peak occurring in December 2007. No further peaks occurred until May 2013. Overall, a two-piecewise temporal trend of measles incidence was observed during the whole 11-year period, i.e. downtrend during 2004-2009 




Fig. 1 Annual trend in measles incidence during 2004-2014 and epidemic curve of monthly measles cases in Guangxi, between January 2004 and December 2014. $\mathbf{a}$ is for trend line, and $\mathbf{b}$ is for epidemic curve

and uptrend during 2010-2014. And a purely temporal cluster of very high incidence was detected in 2014 $(\mathrm{LLR}=1570.73, \mathrm{RR}=3.76, P=0.001)$.

\section{Purely spatial irregular clusters of measles}

Figure 2 shows the spatial interpolation of annual incidence between 2004 and 2014. In general, West, Southwest and North Central Guangxi were the hotspots of measles. Table 1 presents the spatial clusters of high incidence, which are visualized in Fig. 3. The spatial pattern of clustering was consistent with that of incidence interpolation shown in Fig. 2.

\section{Spatio-temporal irregular clusters of measles}

Table 2 summarizes the spatio-temporal clusters of high incidence between 2004 and 2014, which are visualized in Fig. 4. In general, there were three distinct phases of space-time clusters. Specifically, spatiotemporal clusters were identified in Central Guangxi during 2004-2005 (Phase 1), Midwest Guangxi in 2007 (Phase 2), and West and Southwest Guangxi during 2013-2014 (Phase 3). Moreover, a spatio-temporal trend of measles was observed, shifting from Central to West and Southwest Guangxi, which was revealed by linear directional mean as well (Fig. 5).

\section{Patterns of RR and PAR\%}

Figure 6 reveals the spatial patterns of RR and PAR\% among children aged $\leq 24$ months in Guangxi, during 2004-2014. In general, the pattern of RR mostly coincided with that of PAR\%. Regions with high RR and PAR\% were mainly clustered in West, Southwest, and North Central Guangxi. Despite the geographical similarity between the two indicators, heterogeneities in some regions were seen in Xilin, Pingguo, Liangqing, Qingxiu, Ziyuan and Sanjiang counties, with a low or moderate level of RR but high level of PAR\% evident. Thus, a low RR might obscure the fact that some low-risk regions had a heavy burden of measles.

\section{Discussion}

The present study is the first attempt to detect irregular clusters of space, time, and space-time, combining flexibly shaped scan statistics and population attributable risk percent. The findings revealed that a temporal uptrend of measles incidence existed in Guangxi between 2010 and 2014, spatio-temporal clusters of measles had shifted from Central to West and Southwest Guangxi, and regions with high RR and PAR\% were mainly clustered in West, Southwest and North Central Guangxi. These findings not only deepen our understanding of the spatio-temporal patterns of measles in Guangxi, but also assist in identifying regions with high risk and heavy burden of measles, where intensifying surveillance of timeliness and completeness of routine measles vaccination and implementing supplementary immunization activities for measles should be prioritized.

Yellow card alerting system, an innovative vaccination intervention way, works as a core tool for policy intervention to classify counties alerted by a yellow card into EPI blacklist [27]. Since the yellow card alerting system, routine two-dose measles vaccination 


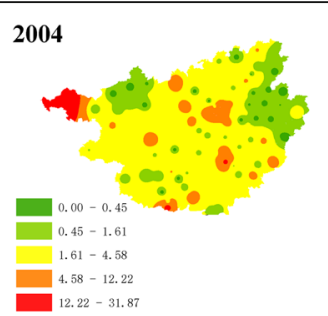

2007



2010

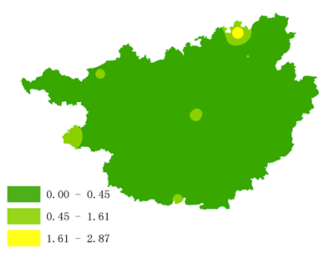

2013

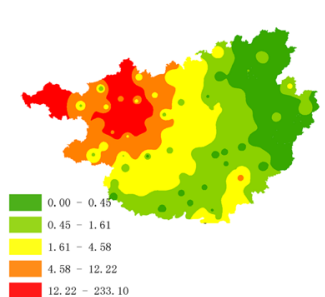

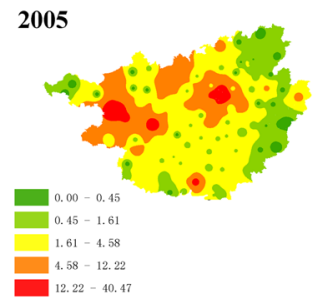

2008



2011

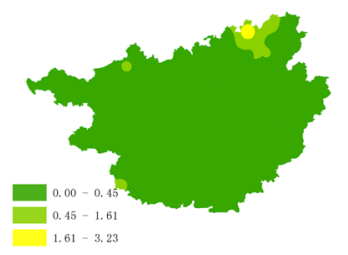

2014
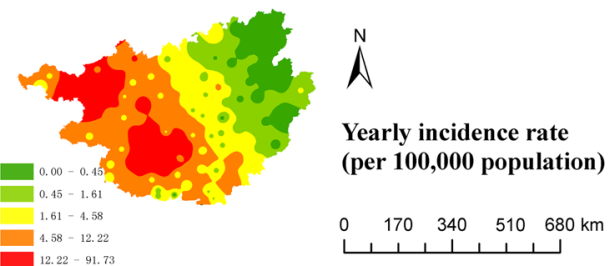
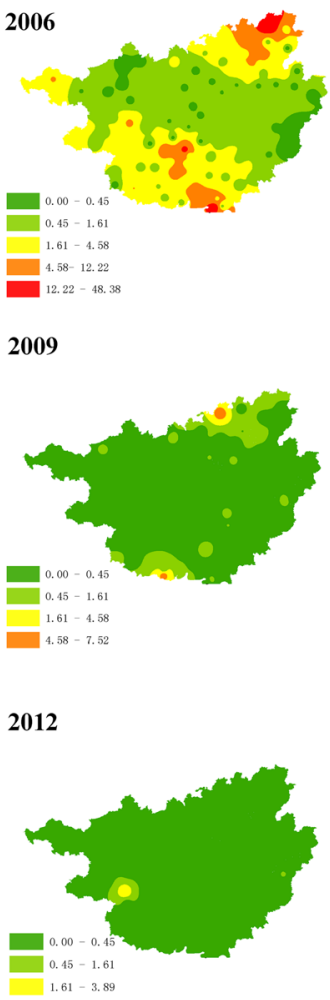

2009

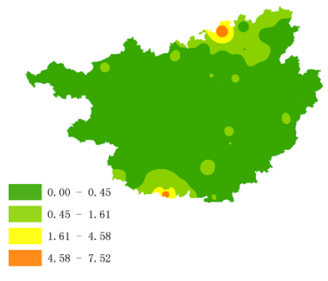

2012

Fig. 2 Spatial interpolation of measles incidence between 2004 and 2014, Guangxi, China

program and supplementary immunization activities for measles were implemented in Guangxi, the measles incidence has remained at a low level [27]. However, largescale outbreaks of measles re-emerged in Guangxi during 2013-2014, possibly attributed to the accumulation effect of a fairly large number of susceptible children in the last decade, which ultimately weakened the herd immunity against measles [28]. The seasonal peaks of measles predominantly occurred between April and June, in addition to a minor peak in December 2007. The minor seasonal peak could be due to the abnormally high temperatures in winter during that year, which created ideal conditions for the spread of measles [29].

Overall, purely spatial clusters of measles constantly emerged in West, Southwest, North and Central Guangxi, though the endemic areas of measles had considerably shrunk after the implementation of the expanded program on immunization. With parents migrating to urban areas for employment opportunities, numerous children were left behind in rural areas, particularly in impoverished and remote areas, where the travel time to vaccination clinics are long and the timeliness and completeness of measles vaccination was low [9]. This might be the reason why the clusters were located in West and North Guangxi, regions with high labour exports. Simultaneously, many migrant children in urban or semi-urban areas did not receive measles vaccination and thus became susceptible to measles [30]. In this light, it was not surprising that clusters of measles occurred in labour-importing regions as well, i.e. Liuzhou in Central and Nanning in Southwest Guangxi.

Regarding the populations vulnerable to nonimmunization, previous studies revealed that both left-behind children and migrant children were the disadvantaged groups in China and suffered from inequities in timely vaccination service $[9,30,31]$. Furthermore, these children have brought great challenges to the 
Table 1 Spatial irregular clusters of annual measles incidence between 2004 and 2014

\begin{tabular}{|c|c|c|c|c|c|c|}
\hline$\overline{\text { Year }}$ & Type of cluster & No. of counties in cluster & Radius (km) & LLR & $\mathrm{RR}$ & $P$-value ${ }^{*}$ \\
\hline \multirow[t]{5}{*}{2004} & Most likely cluster & 2 & 39.17 & 114.21 & 7.12 & 0.001 \\
\hline & Secondary cluster 1 & 8 & 164.99 & 104.56 & 2.76 & 0.001 \\
\hline & Secondary cluster 2 & 6 & 187.76 & 38.18 & 2.06 & 0.001 \\
\hline & Secondary cluster 3 & 4 & 113.48 & 26.68 & 1.75 & 0.001 \\
\hline & Secondary cluster 4 & 3 & 81.03 & 24.37 & 2.66 & 0.001 \\
\hline \multirow[t]{6}{*}{2005} & Most likely cluster & 4 & 26.18 & 381.42 & 7.24 & 0.001 \\
\hline & Secondary cluster 1 & 4 & 117.62 & 355.75 & 6.41 & 0.001 \\
\hline & Secondary cluster 2 & 1 & $0.00^{\mathrm{a}}$ & 85.96 & 5.30 & 0.001 \\
\hline & Secondary cluster 3 & 2 & 61.69 & 35.73 & 2.38 & 0.001 \\
\hline & Secondary cluster 4 & 2 & 64.71 & 28.58 & 2.88 & 0.001 \\
\hline & Secondary cluster 5 & 2 & 53.88 & 9.99 & 2.56 & 0.033 \\
\hline \multirow[t]{4}{*}{2006} & Most likely cluster & 6 & 100.54 & 191.49 & 7.03 & 0.001 \\
\hline & Secondary cluster 1 & 6 & 132.81 & 137.54 & 3.99 & 0.001 \\
\hline & Secondary cluster 2 & 2 & 12.85 & 91.45 & 8.48 & 0.001 \\
\hline & Secondary cluster 3 & 5 & 179.29 & 11.43 & 1.93 & 0.005 \\
\hline \multirow[t]{5}{*}{2007} & Most likely cluster & 1 & $0.00^{\mathrm{a}}$ & 1107.94 & 33.01 & 0.001 \\
\hline & Secondary cluster 1 & 3 & 87.64 & 25.58 & 2.91 & 0.001 \\
\hline & Secondary cluster 2 & 2 & 32.53 & 23.32 & 2.60 & 0.001 \\
\hline & Secondary cluster 3 & 1 & $0.00^{\mathrm{a}}$ & 22.49 & 2.64 & 0.001 \\
\hline & Secondary cluster 4 & 4 & 14.26 & 9.88 & 2.27 & 0.046 \\
\hline \multirow[t]{7}{*}{2008} & Most likely cluster & 4 & 59.44 & 207.67 & 5.14 & 0.001 \\
\hline & Secondary cluster 1 & 4 & 14.26 & 96.97 & 5.97 & 0.001 \\
\hline & Secondary cluster 2 & 1 & $0.00^{\mathrm{a}}$ & 87.52 & 4.93 & 0.001 \\
\hline & Secondary cluster 3 & 4 & 153.81 & 19.88 & 2.52 & 0.001 \\
\hline & Secondary cluster 4 & 1 & $0.00^{\mathrm{a}}$ & 12.49 & 2.56 & 0.002 \\
\hline & Secondary cluster 5 & 1 & $0.00^{\mathrm{a}}$ & 10.57 & 4.64 & 0.018 \\
\hline & Secondary cluster 6 & 1 & $0.00^{\mathrm{a}}$ & 9.62 & 4.93 & 0.050 \\
\hline \multirow[t]{2}{*}{2009} & Most likely cluster & 1 & $0.00^{\mathrm{a}}$ & 66.28 & 33.23 & 0.001 \\
\hline & Secondary cluster 1 & 5 & 136.07 & 20.64 & 4.62 & 0.001 \\
\hline 2010 & Most likely cluster & 1 & $0.00^{\mathrm{a}}$ & 15.51 & 53.67 & 0.002 \\
\hline 2011 & Most likely cluster & 6 & 92.81 & 28.95 & 18.03 & 0.001 \\
\hline 2012 & Most likely cluster & 1 & $0.00^{\mathrm{a}}$ & 42.68 & 56.51 & 0.001 \\
\hline \multirow[t]{4}{*}{2013} & Most likely cluster & 1 & $0.00^{\mathrm{a}}$ & 1924.26 & 81.45 & 0.001 \\
\hline & Secondary cluster 1 & 8 & 205.23 & 163.35 & 4.14 & 0.001 \\
\hline & Secondary cluster 2 & 2 & 49.60 & 18.22 & 2.48 & 0.001 \\
\hline & Secondary cluster 3 & 1 & $0.00^{\mathrm{a}}$ & 13.63 & 2.07 & 0.001 \\
\hline \multirow[t]{3}{*}{2014} & Most likely cluster & 8 & 127.25 & 2006.30 & 5.88 & 0.001 \\
\hline & Secondary cluster 1 & 3 & 71.96 & 431.92 & 7.04 & 0.001 \\
\hline & Secondary cluster 2 & 5 & 153.81 & 24.99 & 1.88 & 0.001 \\
\hline
\end{tabular}

*Tango's flexible scan statistics

aZero-radius means only one county in the cluster

elimination of measles in China. Thus, the shift of spacetime clusters from Central to West and Southwest Guangxi might be partly because of the dynamic changes in demographic structure among children in the above regions.
Although a similar spatial pattern of measles was identified in terms of RR and PAR\%, heterogeneity still existed in Xilin, Pingguo, Liangqing, Qingxiu, Ziyuan and Sanjiang counties. These regions had low or mild 


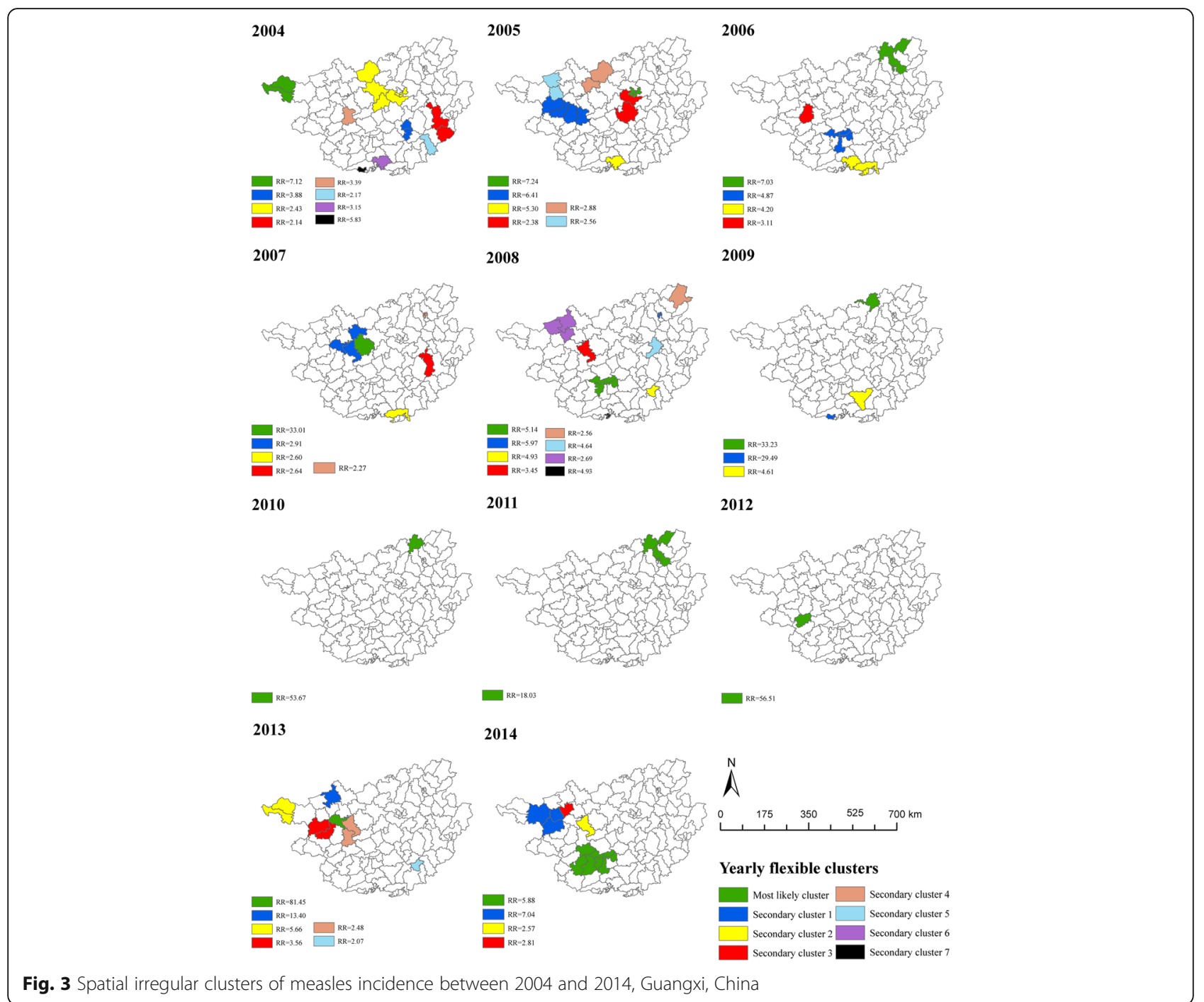

levels of RR but a high level of PAR\%, probably because of the high-density of children aged $\leq 24$ months living there. In China, two doses of measles vaccine should be routinely delivered to children within 24 months after birth [25]. Given the advantage of PAR\% in evaluating the public health significance of preventive measures, the burden of measles would be reduced sharply if routine two-dose measles vaccination and supplementary immunization activities for measles were delivered on time to the age-appropriate children in regions with high
PAR\% [7]. Therefore, combining PAR\% with scan statistics would be a novel approach in the analysis of disease clusters.

The shape of the scanning window is critical for scan statistics and affects the ability to detect clusters of disease accurately and sensitively [32]. So far, numerous studies have applied a circular or cylindrical window to detect regular and compact clusters [11-16]. However, these windows might fail to reflect the true shapes of clusters, due to the fact that clusters, in reality, are

Table 2 Spatio-temporal irregular clusters of annual measles incidence during 2004-2014

\begin{tabular}{lllllll}
\hline Type of cluster & Cluster period & Cluster center & No. of counties & LLR & RR & $P$-value \\
\hline Most likely cluster & 2014 & 108.08 E, 22.92 N & 5 & 1042.05 & 6.22 & 0.001 \\
Secondary cluster 1 & $2013-2014$ & 106.51 E, 24.83 N & 10 & 764.37 & 4.24 \\
Secondary cluster 2 & $2004-2005$ & 109.76 E, 24.02 N & 23 & 637.35 & 4.26 \\
Secondary cluster 3 & 2007 & 108.11 E, 24.17 N & 1 & 552.47 & 9.001 \\
\hline
\end{tabular}

"Kulldorff's space-time scan statistics based on Poisson model and elliptical-cylinder scanning window 




irregular, eccentric and complex [22, 23, 33]. Thus, regularly shaped clusters should be interpreted with caution. To accurately depict the shapes of non-compact clusters and to avoid falsely absorbing neighbouring regions where the actual hazard is not elevated into clusters, we preferred flexibly shaped scan statistics to detect irregular clusters of measles. This is another novelty of the present study.

This study has some limitations. First, the shapes of spatio-temporal clusters were somewhat inflexible. Although the elliptical-cylinder scanning window is more sensitive to clusters that are eccentric, non-compact and noncontiguous in shape than the cylindrical window, it is impossible to completely reflect the true shape of an irregular cluster. Second, the modifiable areal unit problem was inevitable, comprising a zoning effect and a scale effect [34]. On one hand, the adjustment of administrative district at county-level might lead to a zoning effect, which would slightly affect the spatio-temporal patterns of measles. On the other hand, since data at the town-level were not available, the county-level was deemed as the spatial scale to detect clusters. The aggregated data at county-level might obscure the clusters detected at town-level, resulting in the



Fig. 5 Linear directional mean (LDM) during 2004-2014 

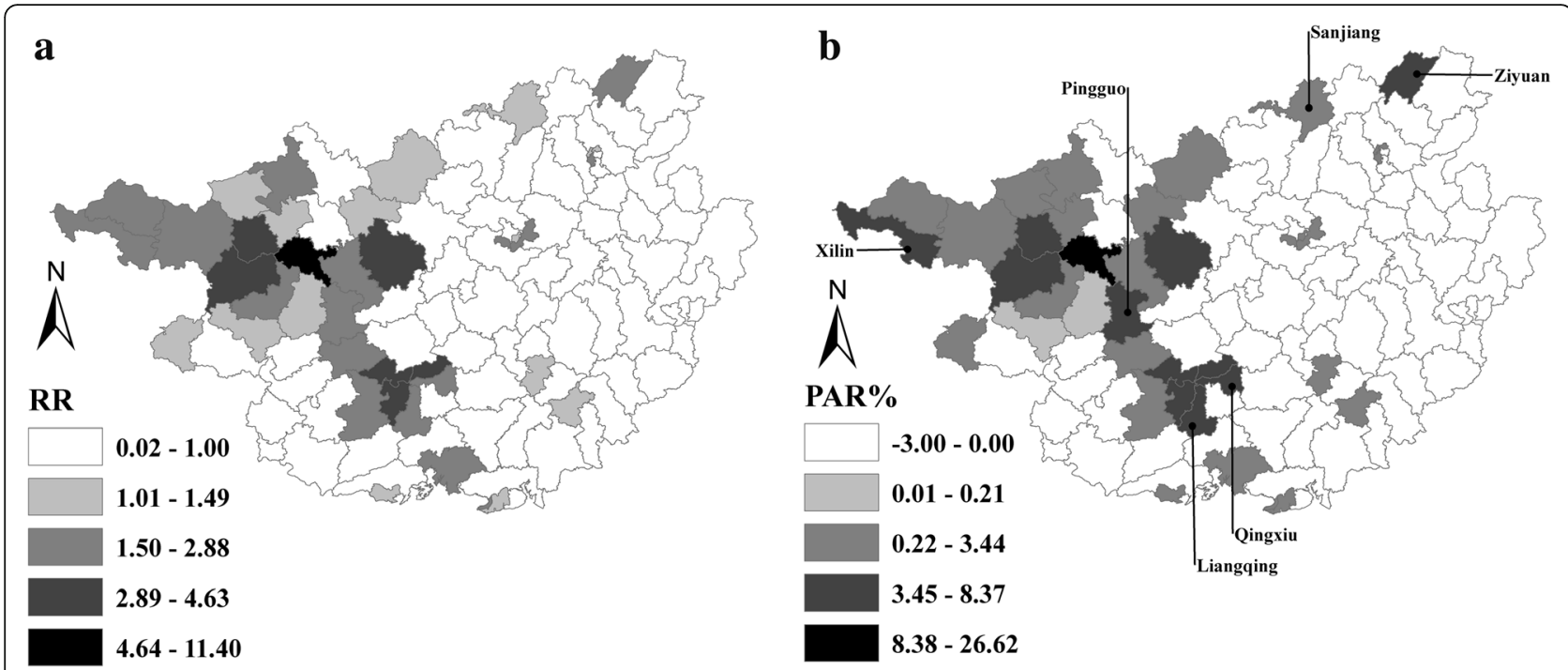

Fig. 6 Spatial patterns of relative risk (RR) and population attributable risk percent (PAR\%) among children aged $\leq 24$ months in Guangxi, $2004-2014$. $\mathbf{a}$ is for $\mathrm{RR}$, and $\mathbf{b}$ is for PAR\%

so called ecological fallacy, which is a sub-problem of scale effect. Third, setting the maximum spatial cluster size of the scanning window is somewhat arbitrary, though we preferred $15 \%$ of population at risk recommended by other studies [22, 23]. Fourth, the accuracy of the flexibly shaped scan statistics was not evaluated in this study [33, 35, 36]. Fifth, PAR\% was underestimated, as we used the proportion of population aged $\leq 24$ months as the percent of exposure to measles, which might not exactly reflect the level of exposure to measles in the whole population. Lastly, factors underlying the spatiotemporal shift trend of measles were not explored quantitatively. Further studies will be conducted to determine the role of socio-economic and demographic factors, geographical barriers and vaccination provider-related determinants in driving the transmission dynamics.

Despite these limitations, our findings have important implications for vaccination policymakers, vaccination practitioners and public health authorities to take essential measures against outbreaks of measles in Guangxi. Given regions with high PAR\%, which were mainly clustered in West, Southwest and North Central Guangxi, supplementary immunization activities for measles should be given priority in these regions. Given the shift of high-risk clusters of measles to West and Southwest Guangxi, a prospective real-time measles surveillance system based on prospective scan statistics should be established in these areas to improve the timeliness and completeness of routine measles vaccination among age-appropriate children. In view of the increasing temporal trend of measles incidence, more measles-related health resources should be allocated in Guangxi than in previous years, particularly in high-risk and heavy-burden regions, and more attention should be paid to develop an early-warning system of measles outbreaks.

\section{Conclusion}

This exploratory study integrated flexibly shaped scan statistics and PAR\% to detect spatial, temporal and spatio-temporal measles clusters in irregular shapes. The findings revealed that there was a temporal uptrend in measles incidence in Guangxi during 20102014, irregular clusters of space-time had shifted from Central to West and Southwest Guangxi, and regions of high risk and heavy burden of measles were mostly concentrated in the West, Southwest and North Central regions of Guangxi. These findings strongly suggest that measures targeting these regions should be developed to prevent future measles outbreaks, and reduce geographical inequities in the burden of measles. Moreover, combination of flexibly shaped scan statistics and PAR\% is a novel approach to improve disease cluster detection, which would be widely used for other similar studies.

\section{Abbreviations}

ASEAN: Association of Southeast Asian Nations; CDC: Center for Disease Control and Prevention; GIS: Geographic Information Systems; LLR: Log Likelihood Ratio; PAR\%: Population Attributable Risk Percent; RR: Relative Risk; WHO: World Health Organization.

\section{Acknowledgments}

This study is part of Xianyan Tang's thesis to fulfill the requirements for PhD degree in Epidemiology at Prince of Songkla University and the China Medical Board under the project of "Join Research Capacity Strengthening of the Western Rural Health Network, China". We would like to thank Yanning Ni at Guizhou University for her assistance in visualizing spatio-temporal clusters in three-dimensions. 


\section{Funding}

This study was funded by the China Medical Board (No. 14-202) and the National Natural Science Foundation of China (No. 81502890). The sponsors had no role in study design, data collection, data analysis, or manuscript writing.

\section{Availability of data and materials}

All data of measles cases used in this study are available from Guangxi Center for Disease Control and Prevention (CDC). Requesting for using the data should be addressed to Guangxi CDC's steering committees.

\section{Authors' contributions}

XYT designed the study, performed the analyses and drafted the manuscript. $A G$ and EM supervised XYT's work regarding spatial-temporal analysis and contributed to optimizing the paper. QYD, AHD and GZ oversaw data collection and helped revise the manuscript. All authors read and approved the final manuscript.

\section{Competing interests}

The authors declare that they have no competing interests.

\section{Consent for publication}

Not applicable.

\section{Ethics approval and consent to participate}

This study was approved by the Ethics Committees of Prince of Songkla University and Guangxi Medical University. Dataset of measles cases was approved by Guangxi Center for Disease Control and Prevention.

\section{Publisher's Note}

Springer Nature remains neutral with regard to jurisdictional claims in published maps and institutional affiliations.

\section{Author details}

'Department of Epidemiology and Biostatistics, School of Public Health, Guangxi Medical University, Guangxi Zhuang Autonomous Region, China. 2Epidemiology Unit, Faculty of Medicine, Prince of Songkla University, Hat Yai, Thailand. ${ }^{3}$ Guangxi Center for Disease Control and Prevention, Institute of Vaccination, Guangxi Zhuang Autonomous Region, China.

Received: 11 November 2016 Accepted: 28 March 2017 Published online: 04 April 2017

\section{References}

1. Peng ZQ, Chen WS, He Q, Peng GW, Wu CG, Xu N, et al. Evaluation of the mass measles vaccination campaign in Guangdong Province, China. Int J Infect Dis. 2012;16(2):e99-e103.

2. Cutts FT, Lessler J, Metcalf CJ. Measles elimination: progress, challenges and implications for rubella control. Expert Rev Vaccines. 2013;12(8):917-32.

3. Ma C, An ZJ, Hao LX, Cairns KL, Zhang Y, Ma J, et al. Progress toward measles elimination in the People's Republic of China, 2000-2009. J Infect Dis. 2011;204(suppl 1):S447-54

4. Chen M, Zhang Y, Huang F, Wang HL, Liu DL, Li J, et al. Endemic and imported measles virus-associated outbreaks among adults, Beijing, China, 2013. Emerg Infect Dis. 2015;21(3):477-9

5. Ma C, Hao LX, Zhang Y, Su Q, Rodewald L, An ZJ, et al. Monitoring progress towards the elimination of measles in China: an analysis of measles surveillance data. Bull World Health Organ. 2014:92:340-7.

6. Wang X, Boulton ML, Montgomery JLP, Carlson B, Zhang Y, Gillespie B, et al. The epidemiology of measles in Tianjin, China, 2005-2014. Vaccine. 2015;33(46):6186-91.

7. Zhu Y, Xu Q, Lin H, Yue D, Song L, Wang C, et al. Spatiotemporal analysis of infant measles using population attributable risk in Shandong Province, 1999-2008. PLOS ONE. 2013;8(11):e79334.

8. Zhuo J, Geng W, Hoekstra EJ, Zhong G, Liang X, Zhang J. Impact of supplementary immunization activities in measles-endemic areas: a case study from Guangxi, China. J Infect Dis. 2011;204(suppl 1):S455-62.

9. Tang X, Geater A, McNeil E, Zhou H, Deng Q, Dong A, et al. Parental migration and children's timely measles vaccination in rural China: a cross-sectional study. Tropical Med Int Health. 2016;21(7):886-94.
10. Kulldorff M. SaTScan user guide for version 9.4. Boston: Department of Ambulatory Care and Prevention, Harvard Medical School; 2015.

11. Carrel M, Emch M, Streatfield PK, Yunus M. Spatio-temporal clustering of cholera: the impact of flood control in Matlab, Bangladesh, 1983-2003. Health Place. 2009;15(3):771-82.

12. Iftimi A, Martínez-Ruiz F, Santiyán AM, Montes F. Spatio-temporal cluster detection of chickenpox in Valencia, Spain in the period 2008-2012. Geospat Health. 2015;10(1):54-62.

13. Jones SG, Conner W, Song B, Gordon D, Jayakaran A. Comparing spatiotemporal clusters of arthropod-borne infections using administrative medical claims and state reported surveillance data. Spat Spatio Temporal Epidemiol. 2012;3(3):205-13.

14. Ling CY, Gruebner O, Krämer A, Lakes T. Spatio-temporal patterns of dengue in Malaysia: combining address and sub-district level. Geospat Health. 2014:9(1):131-40.

15. Liu H, Weng Q, Gaines D. Spatio-temporal analysis of the relationship between WNV dissemination and environmental variables in Indianapolis, USA. Int J Health Geogr. 2008;7:66.

16. Sindato C, Karimuribo ED, Pfeiffer DU, Mboera LEG, Kivaria F, Dautu G, et al. Spatial and temporal pattern of Rift Valley fever outbreaks in Tanzania; 1930 to 2007. PLoS ONE. 2014;9(2):e88897.

17. Costa MA, Kulldorff M. Maximum linkage space-time permutation scan statistics for disease outbreak detection. Int J Health Geogr. 2014;13:20.

18. Zhang WY, Wang LY, Liu YX, Yin WW, Hu WB, Magalhaes RJS, et al. Spatiotemporal transmission dynamics of hemorrhagic fever with renal syndrome in China, 2005-2012. PLoS Negl Trop Dis. 2014;8(11):e3344.

19. Dept. of Immunization, Vaccines and Biologicals, World Health Organization. Manual for the laboratory diagnosis of measles and rubella virus infection. Geneva, 2007

20. Mueller TG, Pusuluri NB, Mathias KK, Cornelius P, Barnhisel R, Shearer S. Map quality for ordinary kriging and inverse distance weighted interpolation. Soil Sci Soc Am J. 2004;68(6):2042-7.

21. Takahashi K, Yokoyama T, Tango T. FleXScan User Guide for version 3.1, 2010. http://www.niph.go.jp/soshiki/gijutsu/index_e.html.

22. Tango T, Takahashi K. A flexibly shaped spatial scan statistic for detecting clusters. Int J Health Geogr. 2005;4:11

23. Kulldorff $M$, Huang L, Pickle L, Duczmal L. An elliptic spatial scan statistic Stat Med. 2006;25(22):3929-43.

24. Rockhill B, Newman B, Weinberg C. Use and misuse of population attributable fractions. Am J Public Health. 1998;88(1):15-9.

25. Zheng J, Zhou Y, Wang H, Liang $X$. The role of the China experts advisory committee on immunization program. Vaccine. 2010;28(suppl 1):A84-7.

26. Samphutthanon R, Tripathi NK, Ninsawat S, Duboz R. Spatio-temporal distribution and hotspots of hand, foot and mouth disease (HFMD) in northern Thailand. Int J Environ Res Public Health. 2013;11(1):312-36.

27. Zhuo J, Hoekstra EJ, Zhong G, Liu W, Zheng Z, Zhang J. Innovative use of surveillance data to harness political will to accelerate measles elimination: experience from Guangxi, China. J Infect Dis. 2011;204(suppl 1):S463-70.

28. E Delaporte, E Jeannot, P Sudre, Wyler Lazarevic C, JL Richard and P Chastonay. Measles in Geneva between 2003 and 2010: persistence of measles outbreaks despite high immunisation coverage. Euro Surveill. 2011;16(39).

29. Lima M. A link between the North Atlantic oscillation and measles dynamics during the vaccination period in England and Wales. Ecol Lett. 2009;12(4):302-14.

30. Hu Y, Li Q, Luo S, Lou L, Qi X, Xie S. Timeliness vaccination of measles containing vaccine and barriers to vaccination among migrant children in East China. PLoS ONE. 2013;8(8):e73264

31. Sun M, Ma R, Zeng $Y$, Luo F, Zhang J, Hou W. Immunization status and risk factors of migrant children in densely populated areas of Beijing, China. vaccine. 2010;28(5):1264-74.

32. M Kulldorff. Spatial scan statistics: models, calculations, and applications. Scan statistics and applications. Springer, Birkhäuser Boston; 1999, p. 303-322.

33. Takahashi K, Kulldorff M, Tango T, Yih K. A flexibly shaped space-time scan statistic for disease outbreak detection and monitoring. Int J Health Geogr. 2008;7:14.

34. Parenteau MP, Sawada MC. The modifiable areal unit problem (MAUP) in the relationship between exposure to $\mathrm{NO}^{2}$ and respiratory health. Int J Health Geogr. 2011;10:58.

35. Duczmal L, Kulldorff M, Huang L. Evaluation of spatial scan statistics for irregularly shaped clusters. J Comput Graph Stat. 2012;15(2):428-42.

36. Read S, Bath P. Willett P, Maheswaran R. Measuring the spatial accuracy of the spatial scan statistic. Spat Spatio-Temporal Epidemiol. 2011;2(2):69-78. 